\title{
Robust Algorithm for Super Resolution and Extracting Noise from DIP using Trimmed Median Filter
}

\author{
Kritika \\ M.Tech. Schlor, Dept. of CSE \\ IET, Alwar, RTU \\ Rajsthan, India
}

\author{
Vedant Rastogi \\ Associate Professor, Dept. of CSE \\ IET, Alwar, RTU \\ Rajsthan, India
}

\begin{abstract}
In the paper the most efficient model to implement super resolution using discrete wavelet transform is shown. The model is a three step process of image registration, interpolation and noise filtering using DWT. The paper resolutions the deduction of noise in the digital gray scale images. Normally, Data, text, picture, can be tainted by an additive noise with the process of the scanning. This method precludes the different type of noise such as Salt and Pepper noise (SP Noise) that reasons black and white spots in the original image. All the process is explained and simulation result is presented to prove the theory.
\end{abstract}

\section{Keywords}

Low Resolution, High resolution, Super resolution, Registration, Interpolation, Restoration, DWT.

\section{INTRODUCTION}

Every digital image has a two-dimensional mathematical representation of digital image. Digital images are made out of pixels i.e. picture component. Every pixel speaks to the dark level for highly contrasting photographs at a solitary point in the image, so a pixel can be spoken to by a small speck of particular shading. The investigation of different clamor display and separating procedures [1], in image preparing, commotion lessening and image rebuilding is relied upon to enhance the subjective examination of an image and the execution criteria of quantitative image investigation systems.

Super resolution [1-6] is one of the topics which are still not made to the textbooks on image and video processing. Super resolution refers to the process of producing a high spatial resolution image from several low resolution images, thereby increasing the maximum spatial frequency and removing the degradations that arise during the image capturing process using a low resolution camera.

[13] Experimental results demonstrate the proposed technique is exceptionally successful to channel salt-and-pepper clamor. The estimation of clamor decrease is troublesome and there is no one of a kind calculation accessible to quantify commotion diminishment of advanced images. So the nature of the commotion lessening in images is measured by the factual amount measures: Root Mean Square Error (RMSE) and Peak Signal-to-Noise Ratio (PSNR). The execution of this channel on images spoiled with various commotions of different clamor levels is contrasted and Wiener separating system [14] Use neural network as the learning algorithm which follows the supervised learning. In this paper Bi-lateral filter is defined for its effectiveness in edge-preserved image Denoising. Bilateral filter improves the De-noising efficiency, preserves the fine structures and also reduces the Rician noise. [15] De-noising an image, different separating strategies are utilized however they have inconsistency between commotion evacuation and Edge protection in an image. This de-noising disagreement has no productive arrangement and they need to define an exchange off between the two opposing perspectives.

\section{BACKGROUND HISTORY}

A sensor with less number of detector elements produced a low resolution image, giving blocky effect. This is because when a scene is photographed with a resolution camera it is sampled at low spatial sampling frequency causing aliasing effect. One could think of reducing the size of photo detector elements there by increasing the density and hence sampling rate. But if the pixel size decreases the amount of light incident on each pixel also decreases and this causes a shot noise which degrades the image quality. With the enhancement of the research and the dynamic price change in the image sensor industries now almost everybody is equipped with a camera. Hence the image processing to improve the image quality will directly impacted by this change. Super resolution [1-6] is one of the topics which are still not made to the textbooks on image and video processing.

\section{METHODOLOGY}

\subsection{Registration}

Image Registration is the procedure of adjusting two or more images of same scene taken at various times, from various perspectives and/or by various sensors [14]. It is a procedure of changing diverse arrangement of information point into one co-ordinate framework. In this paper movement of the image is confined to movements and pivot, so an extremely basic (however precise) methodology is sufficient for image enlistment. All things considered, most of the Registration techniques [9] comprise of the accompanying four stages.

\subsection{Image Restoration and interpolation}

The DWT is computed by successive low pass and high pass filtering of the discrete time domain signal. Its significance is in the manner it connects the continuous tile multi resolution to discrete time filters. In the figure, the signal is denoted by the sequence $x[n]$, where $n$ is an integer. The low pass filter is denoted by $\mathrm{G}_{0}$ while the high pass filter is denoted by $\mathrm{H}_{0}$. At each level, the high pass filter produces detail information of the signal $d[n]$, while the low pass filter with scaling function produces approximations a[n]. 


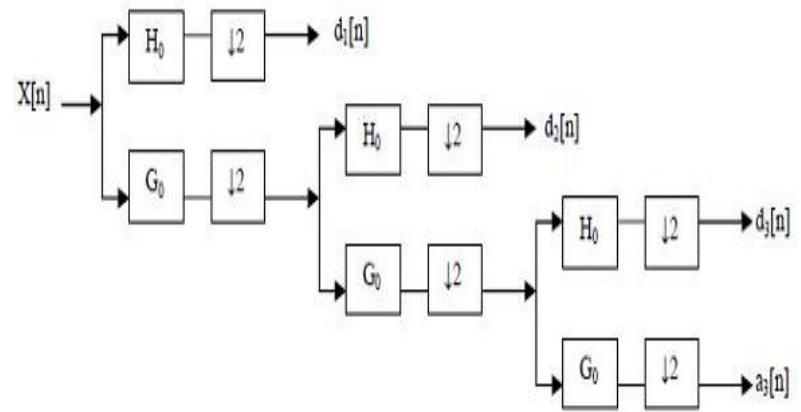

Fig 1: Showing how $\mathrm{i}^{\text {th }}$ pixel of Low resolution image is interpolated onto high resolution grid.

So now according to the modified super resolution diagram, firstly LR images are registered. After registration all the images are interpolated using bilinear interpolation. After that we have $\mathrm{k}$ frames and these are applied to the wavelet transform.

\subsection{A-Trimmed Mean Filter}

In order to calculate the $\alpha$-trimmed mean, the data should be sorted low to high and summed the central part of the ordered array. The number of data values which are dropped from the average is controlled by the trimming parameter $\alpha$ which on the other side of the coin, we already know that the moving average filter suppresses additive white Gaussian noise better than the median filter, while the median filter is better at preserving edges and rejecting impulses. The best choice taking advantages of both moving average and median filter was proposed called the a-trimmed mean filter. The $\alpha$ trimmed mean filter rejects the smaller and the larger observation data depending on the value of $\alpha$. Due to these drawbacks, this dissertation proposed a noble technique which will use improved median filter which gives best performance in restoring images than its existing techniques. We already know that the moving average filter suppresses additive white Gaussian noise better than the median filter

\section{SIMULATION RESULT}

The role of color descriptors has been demonstrated to be quite remarkable in many visual assessment tasks. In some other tasks, texture measurements are needed because of irregularly colored or unusual surfaces. In many applications, color and texture must be combined to achieve good performance. As stated before, we have involved size and shape as well as color and texture. The simulation are performed to discuss super resolution, registration, restoration and transformation technique after this result performed, we are apply salt and pepper noise removal based on nonlocal mean filter technique. So first image will act as reference image and we will convert the second image in to the reference co-ordinate system. Here modified decision based trimmed median filter apply to remove the noise and enhanced the image quality.

\subsection{Registration of an image}

It is a process of aligning two or more images of same scene taken at different times from different view-points. Salient features like closed boundary regions, edges, corners, line intersections and contours are detected automatically.
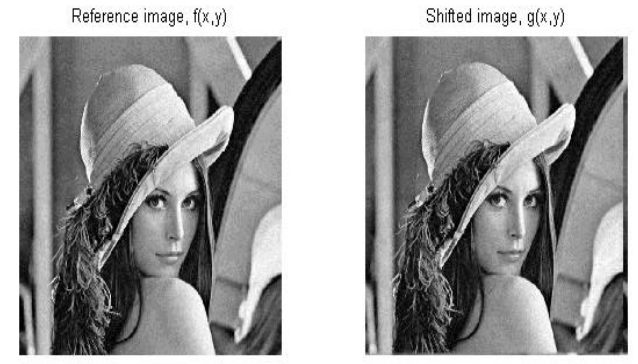

Fig 2: Image shifted based on reference image
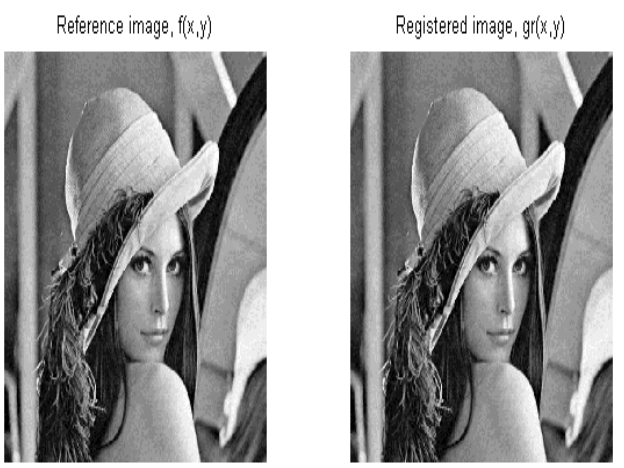

Fig 3: Image registered based on reference image

\subsection{Restoration}

The final super resolution image which is obtain after applying the restoration stage using discrete wavelet transform and fusion algorithm. Here input image is the blurred image and removing the blurred and darkness of the image and get the original and high resolution pixel image.

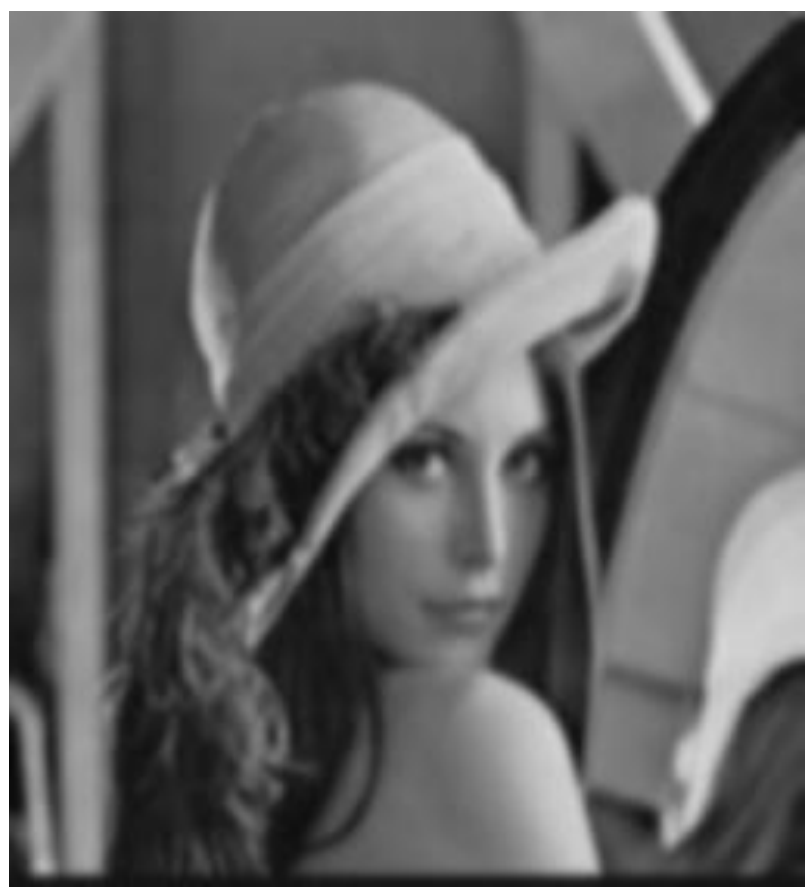

Fig 4: Image used for restoration 


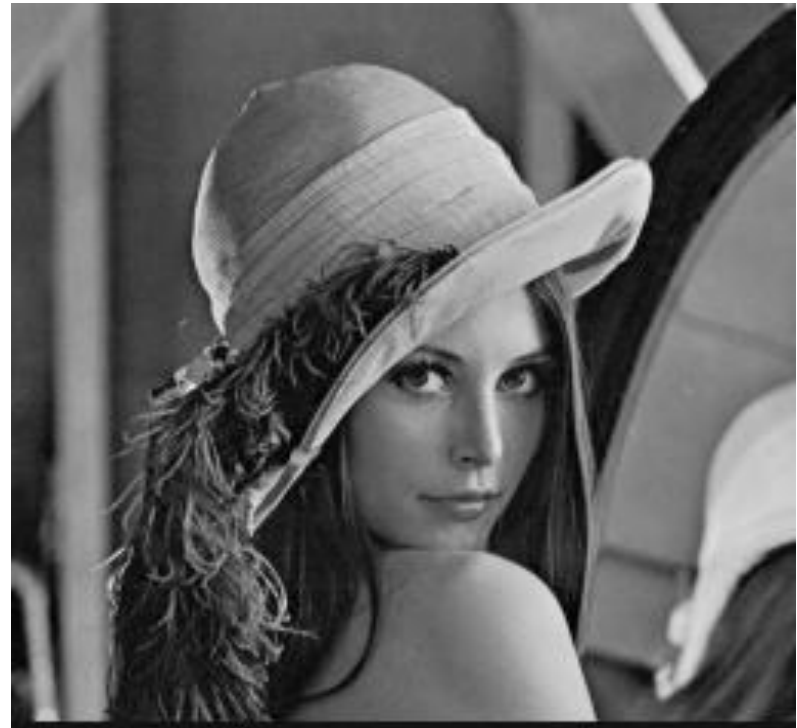

Fig 5: Restored Image

\subsection{Interpolation}

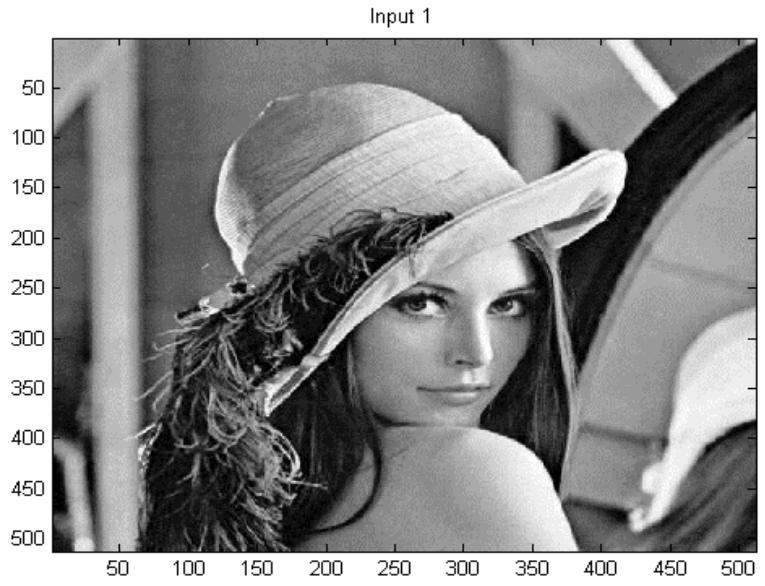

Fig 6: Image used for interpolation

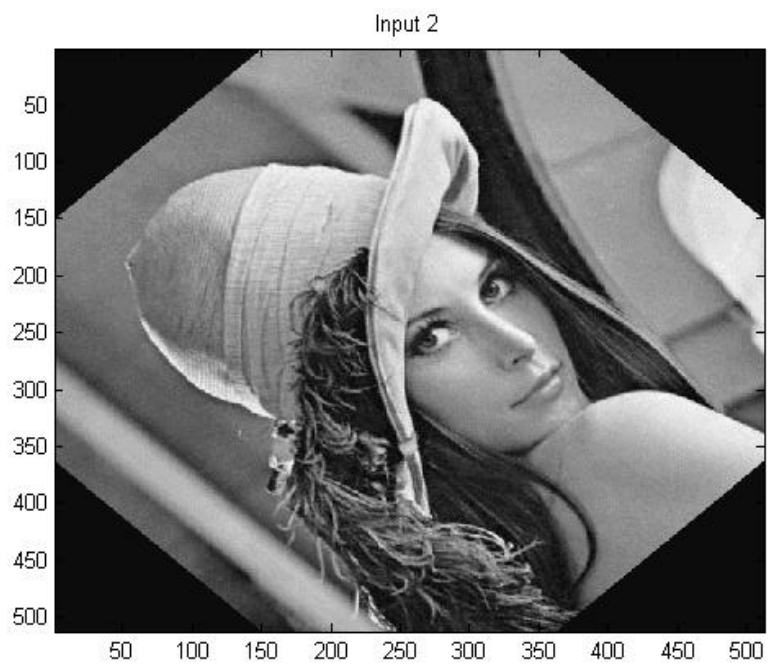

Fig 7: Interpolated Image

\subsection{Salt and Pepper Noise Removal}

The purpose of calculating the performance of the image and after that comparison between then, will show which image are better for noise removing. Such method is mainly due to highly accurate noise detection experienced by the noise detection algorithm having high noise detection ratio and our method performs more desirable than the median filter and other conventional edge preserving method. The (Peak signal to noise ratio) PSNR, (Signal to noise ratio) SNR is high; (mean squared error) MSE is low. This advised method is a fast method for removing salt and pepper noise. The (MSE) of an estimator procedures the average of the squares of the errors that is, the difference between the estimator and what is estimated. MSE is a threat function, corresponding to the expected value of the squared error loss or quadratic loss. The term signal to noise ratio (PSNR) is an expression for the proportion between the most extreme conceivable estimation of a sign and the force of mutilating commotion that influences the nature of its representation. Since numerous signs have a wide element run, the PSNR is generally communicated regarding the logarithmic decibel scale. Image improvement or enhancing the visual nature of a computerized image can be subjective. Saying that one strategy gives a superior quality image could differ from individual to individual. Therefore, it is important to build up quantitative/observational measures to think about the impacts of image upgrade calculations on image quality.

Utilizing the same arrangement of tests image, distinctive image up grade calculations can be contrasted deliberately with distinguish whether a specific calculation creates better results. The metric under scrutiny is the signal to noise ratio. In the event that we can demonstrate that a calculation or set of calculations can improve a corrupted known picture to all the more nearly look like the first, then we can all the more precisely presume that it is a superior calculation. The given table demonstrates the complete execution of the image as indicated by the table topical channel is the best channel for clamor removable procedure.

$$
\begin{gathered}
\text { PSNR in } d B=10 \log _{10}\left(\frac{255^{2}}{M S E}\right) \\
M S E=\frac{\sum_{i} \sum_{j}\left(\gamma(i, j)-\gamma(i . j)^{2}\right)}{M \times N}
\end{gathered}
$$

Table 1. Comparison Table of PSNR, IEF and MSE

\begin{tabular}{|c|c|c|c|c|}
\hline SR NO & Noise in \% & $\begin{array}{c}\text { PSNR IN } \\
\text { DB }\end{array}$ & MSE & IEF \\
\hline $\mathbf{1}$ & 10 & 17.7245 & 0.0169 & 1.8574 \\
\hline $\mathbf{2}$ & 20 & 14.7819 & 0.0333 & 1.8620 \\
\hline $\mathbf{3}$ & 30 & 12.9347 & 0.0509 & 1.8244 \\
\hline $\mathbf{4}$ & 40 & 11.3800 & 0.0728 & 1.7274 \\
\hline $\mathbf{5}$ & 50 & 10.0516 & 0.0988 & 1.5821 \\
\hline $\mathbf{6}$ & 60 & 8.7243 & 0.1341 & 1.3980 \\
\hline $\mathbf{7}$ & 70 & 7.4614 & 0.1794 & 1.2228 \\
\hline $\mathbf{8}$ & 80 & 6.3235 & 0.2332 & 1.0741 \\
\hline $\mathbf{9}$ & 90 & 5.4074 & 0.2879 & 0.9790 \\
\hline
\end{tabular}




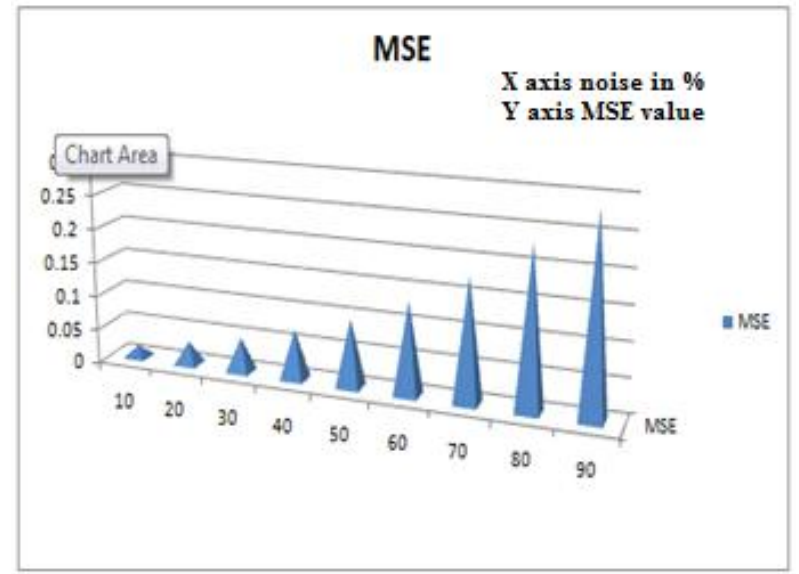

Fig 8: Triangular analysis of MSE with Noise

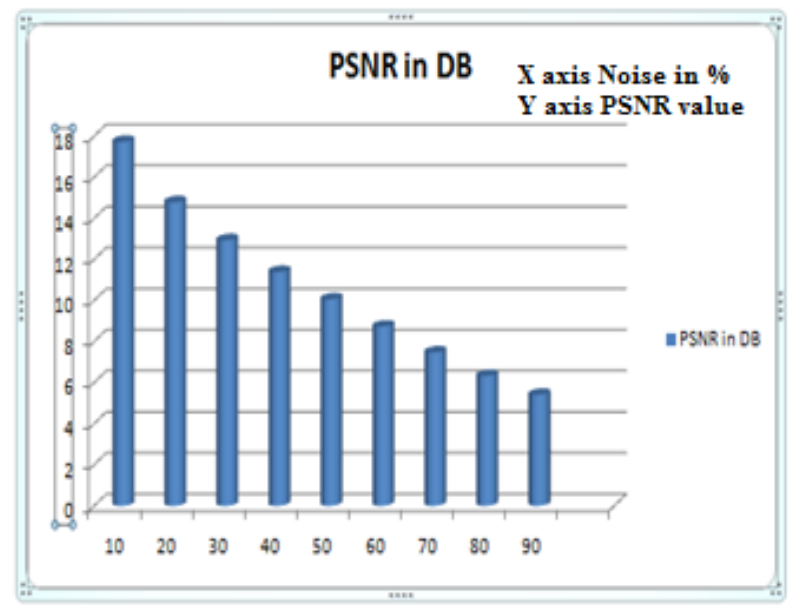

Fig 9: Bar graph of PSNR with Noise

When we added $50 \%$ salt and pepper noise in an image we got following recovered images as depicted below

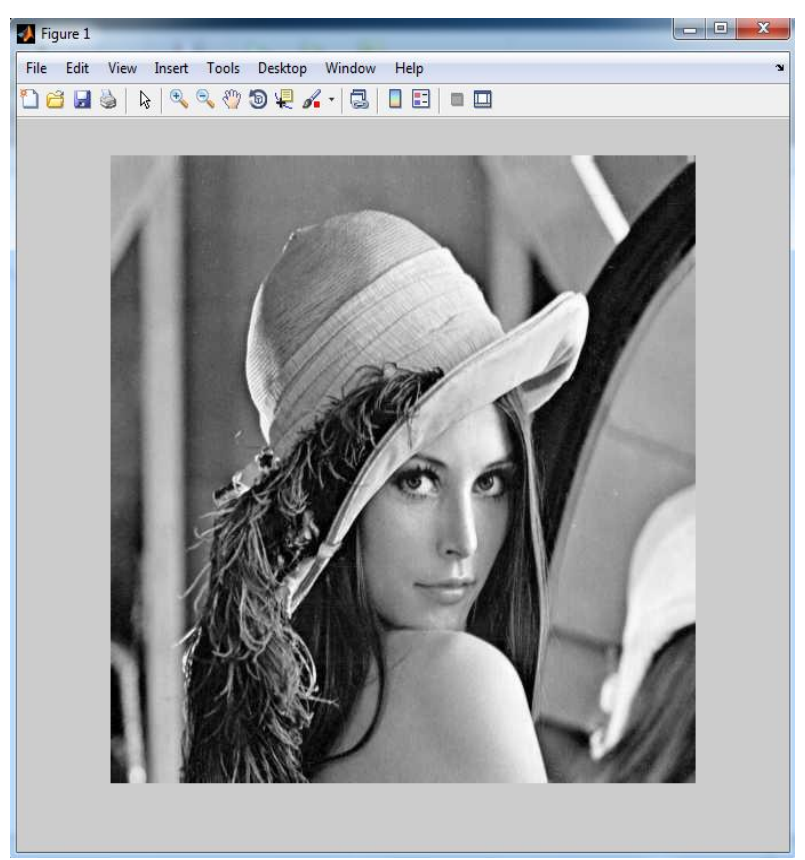

Fig 10: Original image before salt and pepper noise

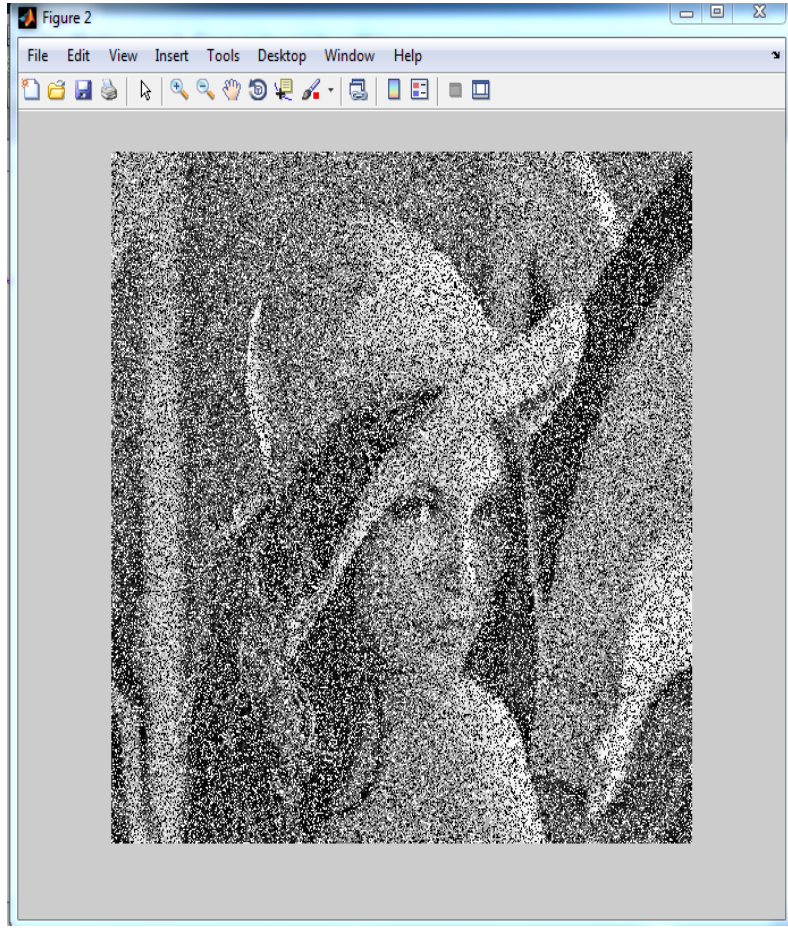

Fig 11: Original Image with 50\% noise

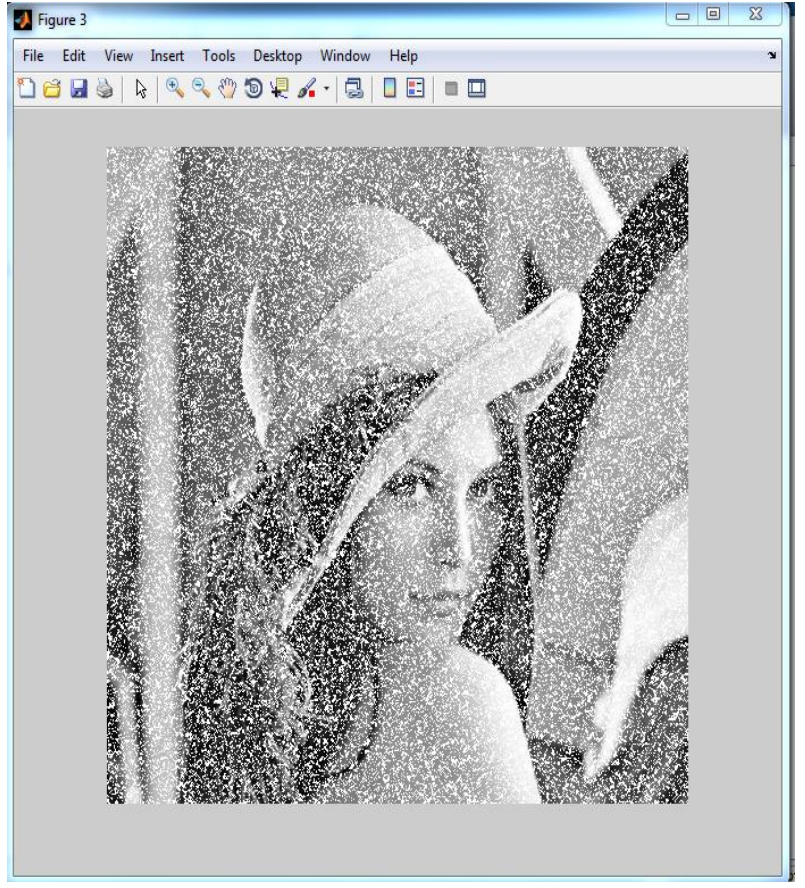

Fig 12: Original Image recovered with $50 \%$ noise

Now we take different images at a particular noise level and calculate different parameter. Every image consist different information so all the parameters will be different. Even for a single image but for different format like TIFF. BMP, JPEG and PNG, PSNR IEF and MSE will be different. These parameters will show high PSNR and IEF; MSE will show less. So, this method is a robust and optimised method for removing salt and pepper noise from an image because it combines median filter and moving average filter algorithms. This method takes advantages from both algorithms. 
Table 2. Comparison Table of PSNR, IEF and MSE

\begin{tabular}{|l|l|l|l|}
\hline Image Name & MSE & PSNR & IEF \\
\hline Angelina & $\mathbf{0 . 0 6 5 7}$ & 11.8264 & 1.1908 \\
\hline Obama & $\mathbf{0 . 0 5 8 8}$ & 12.5356 & 1.2308 \\
\hline Modi & $\mathbf{0 . 0 1 6 1}$ & 17.9264 & 4.880 \\
\hline College & $\mathbf{0 . 0 1 9 4}$ & 17.115 & 3.5650 \\
\hline Bk Hawklac & $\mathbf{0 . 0 1 7 3}$ & 17.6079 & 3.4961 \\
\hline
\end{tabular}

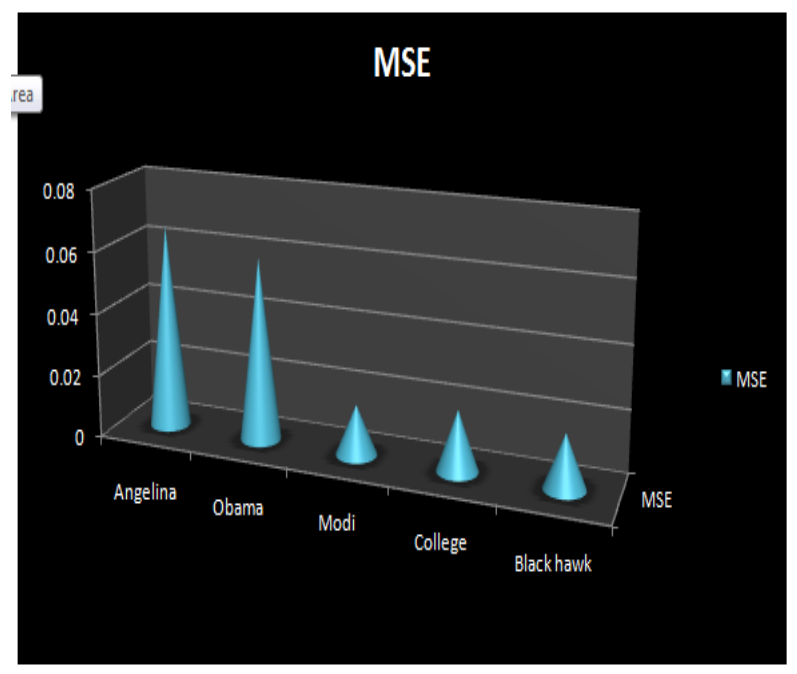

Fig 13: Value of MSE for different image with Noise $20 \%$

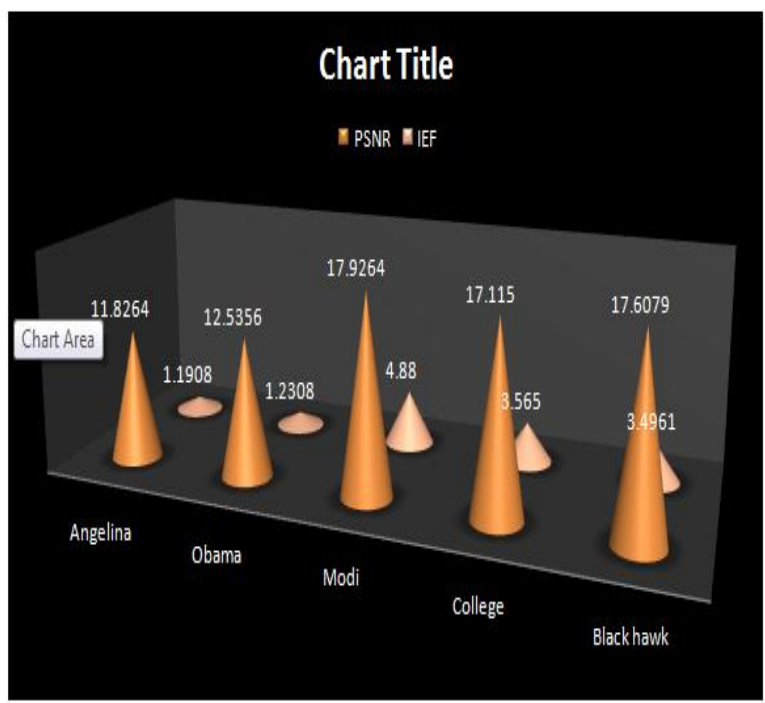

Fig 14: Value of PSNR and IEF for different image with Noise 20\%
Table 3. Comparison Table of PSNR and IEF

\begin{tabular}{|l|r|r|}
\hline Image Format & \multicolumn{1}{l|}{ PSNR } & \multicolumn{1}{l|}{ IEF } \\
\hline College.jpg & $\mathbf{2 0 . 2 4 5 9}$ & $\mathbf{3 . 6 7 5 4}$ \\
\hline College.png & $\mathbf{1 9 . 9 3 8 8}$ & 3.4663 \\
\hline College.bmp & $\mathbf{2 0 . 2 1 5 0}$ & 3.6159 \\
\hline College.gif & $\mathbf{2 0 . 3 4 5 9}$ & 3.5459 \\
\hline
\end{tabular}

\section{CONCLUSION}

Our main focus is to remove salt and pepper noise from an image. As we know when we take a snap from a device and then transmit it through channel or electromagnetic waves automatically noise added. There are various types of noise and to remove noise we have to use filter of various type. Every filter has its own characteristics that why we have to select appropriate filter to remove noise from image. In our work we remove salt and pepper noise with help of alpha trimmed median filter. Besides this we also calculated MSE (Mean Square Error), PSNR (Peak signal to noise ratio) and IEF (Image enhancement factor. We applied our algorithm on various image like color image which include JPEG, PNG, BMP and TIFF format images and we also applied on gray images and find out MSE (Mean Square Error), PSNR (Peak signal to noise ratio) and IEF (Image enhancement factor). After that we analyze our result. Our algorithm is so strong that even if we add noise up to $90 \%$ then it can also able to find out valuable information from the image. After that we draw a lot of graph to depict our result in a simple way. So, we recommend an algorithm for eliminate salt and pepper noise removal.

In the paper an improved super resolution model is proposed by modifying the restoration stage. All the stage is discussed properly with improved restoration stage. The simulation result is performed to check the theory and can be seen that the final image is an improved result. And there is lot of improvement in the super resolution model to improve the further quality of image should be numbered and flush left. For a section head and a subsection head together (such as Section 3 and subsection 3.1), use no additional space above the subsection head.

\section{REFERENCES}

[1] R.Y. Tsai, T.S. Huang, "Multi-frame image restoration and registration", Advances in computer vision and image processing, Vol. 1(2), 1984, pp.317-339

[2] S.C. Park, M.K. Park and M.G. Kang "Super Resolution and Image Reconstruction: A Technical Overview", IEEE Signal Processing Magazine 3(20),21-36(2003)

[3] T Komatsu, K Aizawa, T Igarashi, and T. Saito, "Signal processing based method for acquiring very high resolution image with multiple cameras and its theoretical analysis," Proc. Inst. Elec. Eng., Vol. 140 no. 1, pt. I, pp 19-25Feb1993. 
[4] S. Chaudhuri, Ed, Super-Resolution Imaging. Norwell, MA: Kluwer, 2001. H. Ur and D. Gross, "Improved resolution from sub-pixel shifted pictures," CVGIP: Graphical Models and Image Processing, Vol. 54, pp. 181-186, Mar, 1992.

[5] T. Komatsu, T. Igarashi, K. Aizawa, and T. Saito, "Very high resolution imaging scheme with multiple differentaperture cameras," Sinal Processing: Image Commun., vol. 5, pp. 511-526, Dec. 1993.

[6] P.Kamboj, V.Rani, Image Enhancement Using Hybrid Filtering Technique, IJSR, Vol. 2(6), 2013, 214-220.

[7] Wang, Changhong, Taoyi Chen, and Zhenshen Qu. "A novel improved median filter for salt-and-pepper noise from highly corrupted images." In Systems and Control in Aeronautics and Astronautics (ISSCAA), 2010 3rd International Symposium on, pp. 718-722. IEEE, 2010.

[8] Zhu, Rong, and Yong Wang. "Application of Improved Median Filter on Image Processing." Journal of Computers 7, no. 4 (2012): 838-841.

[9] Tripathi, A. K., and S. Mukhopadhyay. "Single image fog removal using bilateral filter." In Signal Processing, Computing and Control (ISPCC), 2012 IEEE International Conference on, pp. 1-6. IEEE, 2012.
[10] Jayaraj, V., D. Ebenezer, and K. Aiswarya. "High density salt and pepper noise removal in images using improved adaptive statistics estimation filter." International Journal of Computer Science and Network Security 9.11 (2009): 170-176.

[11] Al-amri, Salem Saleh, Namdeo V. Kalyankar, and Santosh D. Khamitkar. "A comparative study of removal noise from remote sensing image." arXiv preprint arXiv: 1002.1148 (2010)

[12] P.Kamboj, V.Rani, Image Enhancement Using Hybrid Filtering Technique, IJSR, vol. 2(6), 2013, 214-220.

[13] R.H.Chan, C.W. Ho, M.Nikolova, Salt-and-Pepper Noise Removal by Median-Type Noise Detectors and DetailPreserving Regularization, IEEE Transactions on Image Processing, vol. 14, No. 10, 1479-1485, 2005.

[14] Nair, M.S., Revathy, K.; Tatavarti, Removal of Salt and Pepper Noise in Images: A new Decision based Algorithm, IMECS'08, and vol. 1, 2008.

[15] B.Singh, R.Singh, H.Singh, Removal of High Density Salt \& Pepper Noise in Noisy color Images using Proposed Median Filter, vol 2, No 2, 2013. 